\title{
"DEPENDENCE OF DYNAMIC ELECTROKINETIC-COUPLING-COEFFICIENT ON THE ELECTRIC DOUBLE LAYER THICKNESS OF FLUID-FILLED POROUS FORMATIONS,
}

\author{
Peng Shi ${ }^{1}$, Wei Guan ${ }^{1}$ and Hengshan $\mathrm{Hu}^{1}$ \\ (1) Department of Mechanics, Harbin Institute of Technology, Harbin, P. R. China
}

Article history

Receveid August 25, 2017; accepted April 2, 2018.

Subject classification:

Electrokinetic coupling coefficient; Porous media; Streaming current; Electroosmosis; Electrical double layer.

\begin{abstract}
Electrokinetic coupling between pore-fluid flow and electric field arising from the electrical double layer (EDL) has many applications in geoscience. In this study, we extended the formulas for the dynamic electrokinetic coupling coefficient (ECC) to arbitrary scaled capillaries. These two ECC formulas for the cylindrical and slit apertures, respectively, were derived without the thin or thick EDL assumption used in previous studies, relating to the normalized radius (the ratio of capillary radius to Debye length). By the identical ECC formulas for streaming current and electroosmosis effects, it is confirmed that Onsager's reciprocity is generally satisfied for arbitrary scaled and shaped apertures. This ECC tends to the results using the thick and the thin EDL assumptions respectively with the decrease and increase of the normalized radius. It is shown that the relative error is less than $10.0 \%$ if the normalized radius is less than 0.8 and is larger than 20, respectively. Otherwise, the thick and the thin EDL assumptions are inapplicable. The high-frequency limit phase of this ECC increases from $45^{\circ}$ to $90^{\circ}$ with the decrease of the normalized radius, rather than that remains at $45^{\circ}$ under the thin EDL assumption. The linear approximation for solving the Poisson-Boltzmann equation influences the electric potential in the EDL and the ECC, which increases with the decrease of the normalized radius. If the normalized radius is larger than 7, the error is within 5.0\% even though the linear approximation has been mathematically invalid when the salinity is $0.001 \mathrm{~mol} / \mathrm{L}$ and zeta potential is $-150 \mathrm{mV}$.
\end{abstract}

\section{INTRODUCTION}

Pore-fluid flow and electric field are coupled in fluid-filled porous media due to the electrical double layer (EDL) with excess charges. As shown in Figure 1, the pore fluid nearby fluid-solid interface is countercharged with excess cations because of the negatively charged surface of the solid frame. When the pore fluid is mechanically forced to flow relatively to the solid frame, some of the excess charges are dragged to move, thereby causing streaming electric current [Quincke, 1861]. Conversely, an applied electric field forces the excess charges to move, thereby driving pore fluid flow, which is referred to as the electroosmosis effect [Wiede- mann, 1852]. These electrokinetic phenomena have various applications in geophysical exploration [e.g. Hu et al., 2000, 2002; Dupuis et al., 2009; Sava and Revil, 2012; Guan et al., 2013, 2015; Monetti et al., 2014; Zyserman et al., 2015], hydrogeophysics [e.g. Warden et al., 2013; Kröger et al., 2014; Ozaki et al., 2014; Rangarajan et al., 2014; Desroches and Butler, 2016] and earthquake warning [e.g. Ishido and Mizutani, 1981; Pride et al., 2004; Gao et al., 2013, 2016]. More introductions to electrokinetic phenomena and their applications in geosciences are available in some tutorials [e.g. Jouniaux and Zyserman, 2016].

Theoretical research of electrokinetic effects can be traced back to the well-known Helmholtz-Smolu- 


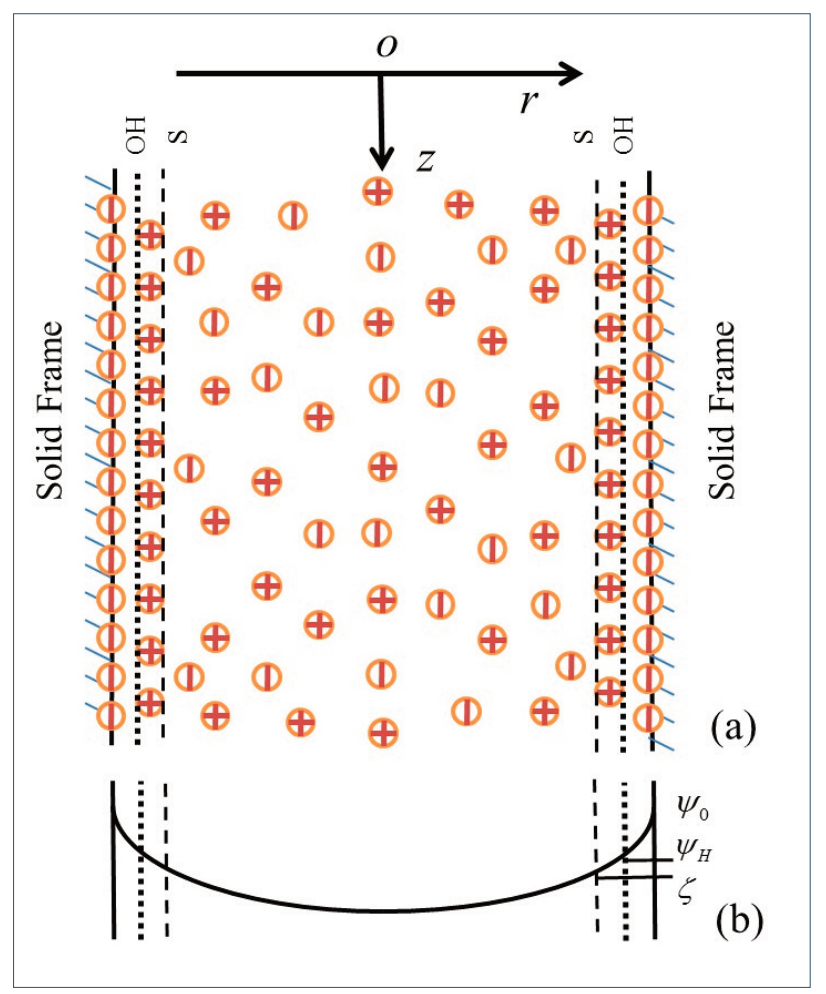

FIGURE 1. Schematic view of Stern's electrical double layer (EDL) model. (a) Charge distribution. (b) Electric potential distribution.

chowski (H-S) equation, first proposed by Von Helmholtz [1879] and then discussed by Smoluchowski [1905] systematically. Subsequently, the EDL model was provided by Gouy [1910] to address the origin of the excess charges and characterize the charge distribution. Based on the H-S equation and the EDL model, many experimental and theoretical attempts have been done to study the electrokinetic phenomena in various fields [e.g. Gaudin and Fuerstenau, 1955; Li and De Bruyn, 1966; Cases, 1969; Somasundaran and Kulkarni, 1973; Reppert and Morgan, 2003; Bordes et al., 2008; Allègre et al., 2012; Wang et al., 2015]. The original H-S equation $\left(L_{0}=\varepsilon \xi / \eta\right.$, where $\varepsilon$ is the dielectric permittivity of fluid, $\zeta$ is the zeta potential, $\eta$ is the fluid viscosity) was proposed for the electroosmosis effects in capillaries to characterize the ratio of the fluid-flow velocity to the applied electric field. On this basis, it was extended to the streaming current/potential effects in capillaries. The modified H-S equations are collectively referred to as the electrokinetic coupling coefficient (ECC). Specifically, it is termed the streaming potential coefficient for the ratio of the electric-potential difference (electric voltage) to the applied pressure difference, and is termed the electroosmosis pressure coefficient for the ratio of the pressure difference to the applied electric-potential difference [Pengra et al., 1999]. The H-S equation is also extended to porous rocks straightforwardly by
$L_{0}=\varphi \varepsilon \zeta / \tau \eta$ [Pride, 1994], where $\varphi$ and $\tau$ denote the porosity and tortuosity, or by other ones with respect to the permeability, the particle size of the solid-frame, the pore diameter, or the pore-throat size [e.g. Li et al., 1995; Glover and Walker, 2008; Glover and Déry, 2010].

Experiments have shown that the ECC changes with frequency [Tardif et al., 2011; Jouniaux and Bordes, 2012]. For time-varying electrokinetic phenomena such as those generated by elastic waves, the H-S equation should be extended to frequency-dependent versions. Three kinds of frequency-dependent ECCs were provided [Jouniaux and Bordes, 2012], which are the second-order vibrational system model [Thomson, 1996], Packard's model for capillaries [Packard, 1953; Reppert et al., 2001] and Pride's model for fluid-saturated porous media based on first principles [Pride, 1994; Walker and Glover, 2010]. These three frequency-dependent ECCs all originate from the $\mathrm{H}-\mathrm{S}$ equation. At the low-frequency limit, the Packard's ECC is exactly the classic H-S equation $L_{0}=\varepsilon \zeta / \eta$, and the Pride's ECC degenerates to the modified $\mathrm{H}-\mathrm{S}$ equation $L_{0}=\varphi \varepsilon \zeta / \tau \eta$. According to Tardif et al. [2011], all the three models have the same trend with the experimentally measured data for Ottawa sandstones, and moreover the pore radii can be accurately estimated from the Packard's ECC. This indicates the rationality of using the H-S equation to characterize various electrokinetic effects in fluidfilled porous media.

The classic H-S equation and the frequency-dependent ECC models mentioned above are valid only when the capillary radius or the pore size is much larger than the EDL thickness. In this case, the excess charges are concentrated nearby the solid-fluid interface, the electric potential changes sharply from the zeta potential at the shear-slip surface to zero, and thus the ECC is dominated by the zeta potential. Such a thin EDL assumption, however, is not always satisfied, especially for natural or artificial porous media with low permeabilities, such as mudstone, shale and concrete. According to Jackson and Leinov [2012], the thin EDL assumption may be not reasonable even for sandstones (having higher permeability whose pore radii are generally larger than $10^{3} \mathrm{~nm}$ ) if the salinities are lower than 0.001 $\mathrm{mol} / \mathrm{L}$. Thus, it is necessary to break the limit of the thin EDL assumption and extend the frequency-dependent ECC model to arbitrary capillary/pore scales and salinities. Burgreen and Nakache [1964] and Rice and Whitehead [1965] derived the analytical solutions of the electric potential and electrokinetic flow velocity in capillary silt and cylindrical capillary with arbitrary scales, respectively. However, neither of them investigated the influence of the capillary scale on the ECC. 
Recently, Fiorentino et al. [2016, 2017] analyzed the effect of the capillary scale on the streaming potential coefficient by using the Lattice Boltzmann method, but the frequency dependence was not involved and the analytical expression was not obtained. Although Revil and Mahardika [2013] provided a frequency-dependent ECC formula for arbitrary pore sizes, their model is not based on the H-S equation in which they employed the effective charge density rather than the zeta potential that widely adopted in previous studies.

In this study, we derive the formula of the frequency-dependent ECC model for arbitrary capillary/pore scale by using the H-S equation. To investigate whether the Onsager's reciprocity [Onsager, 1931] is still valid for arbitrary scale, we consider both the streaming current and electroosmosis effects. Besides, we analyze the influences of the Debye-Hückel approximation [e.g. Pride and Morgan, 1991] on the electric potential and the ECC by comparing the analytical and numerical results. The Debye-Hückel approximation is a linear approximation that is inevitable to be used for the analytical solutions of the PoissonBoltzmann equation.

The rest of this paper is organized as follows. In Section 2.1, we briefly restate the Poisson-Boltzmann equation for the charge distribution and the Navier-Stokes equation for the pore-fluid flow. In Section 2.2, we derive the ECC formulas for a cylindrical capillary and a capillary slit, respectively, according to the streaming current effect. And then the ECC formulas according to the electroosmosis effect are derived in Section 2.3. From these ECC formulas, we check the Onsager's reciprocity. In Section 3.1, we investigate the characteristics of the static and frequency-dependent ECCs for capillaries with arbitrary scale and shape and give comparisons between our ECC model and the ones under the thin and thick EDL assumptions, respectively. After that, we analyze the influences of the Debye-Hückel approximation on the electric potential and the ECC in Section 3.2. Finally, we will derive the main conclusions.

\section{FORMULATION}

\subsection{Basic theory and equations}

Figure 1 shows Stern's EDL model [e.g. Pride and Morgan, 1991], where there are excess charges in the EDL composed of the Stern and the diffuse layers. Suppose that all the cations in the Stern layer are firmed and they don't give any contribution to electrokinetic conversions. For the axisymmetric cylindrical coordi- nate system $(r, z)$, whose origin coincides with the crosssection centroid of the capillary, the distribution of the excess charges in the diffuse layer is governed by the Poisson-Boltzmann equation [Gierst, 1966],

$$
\nabla \cdot(\varepsilon \nabla \psi(r))=-\rho_{e}(r)
$$

where

1. $\rho_{\mathrm{e}}(\mathrm{r})=-2 e v n^{0} \sinh \left(v e \psi(\mathrm{r}) / k_{b} T\right)$ is the charge density,

2. $\psi(\mathrm{r})$ denotes the electric potential varying with coordinate $r$,

3. $\varepsilon$ is the dielectric permittivity which is treated as a constant in this study,

4. $v, n^{0}$ and $T$ are ionic valence, ionic number and kelvin temperature, respectively,

5. $e=1.6 \times 10^{-19} \mathrm{C}$ is the electron charge

6. and $k_{b}=1.3810 \times 10^{-23}$ is the Boltzmann constant.

The following two boundary conditions are also needed to solve Equation (1),

$$
\psi(R)=\zeta,\left(\frac{\partial \psi(r)}{\partial r}\right)_{r=0}=0
$$

where $r=R$ is at the shear-slip surface as shown in Figure 1 .

Considering the electrolyte solution as a Newtonian fluid, the flow is governed by the Navier-Stokes equation,

$$
\rho \frac{\partial u(r)}{\partial t}=\eta\left(\frac{\partial^{2}}{\partial r^{2}}+\frac{1}{r} \frac{\partial}{\partial r}\right) u(r)-\frac{\partial P}{\partial z}+\rho_{e}(r) E
$$

where $u(r)$ is the flow velocity dependent on the coordinate $r, t$ is time, $P$ and $E$ are the fluid pressure and the electric field in the capillary, respectively, $\rho$ and $\eta$ are density and viscosity of the fluid, respectively. The second and third terms on the right side of Equation (3) denote the pressure gradient and the electric field force applied to the fluid, respectively. According to Equation (3), the fluid flow can be caused by both the pressure difference and the electric field due to the electrokinetic effect.

\subsection{Streaming-current ECC}

In this subsection, we derive the frequency-dependent ECC for the streaming current effect caused by an applied pressure difference along the capillary axis. In this case, the third term on the right side of Equation (3) vanishes. To solve Equation (3) without $\rho_{\mathrm{e}}(r) E$ and apply the boundary condition $u(r)=0$, we can obtain the fluid flow velocity $u(r)$ driven by the pressure difference.

The solution of $u(r)$ depends on the cross section of 
the capillary. Different-shaped capillaries have different velocity distributions along the $r$-direction. In this study, we consider two typical-shaped capillaries, which are the cylindrical capillary and the capillary slit, to idealize the circular aperture and the flat crack in porous media, respectively. For the cylindrical capillary, the fluid velocity driven by a sinusoidal pressure gradient $\partial P / \partial \mathrm{z}=-A \exp (i \omega t)$ is,

$$
u(r)=\frac{i A}{\omega \rho}\left(\frac{I_{0}(\kappa r)}{I_{0}(\kappa R)}-1\right) \exp (i \omega t)
$$

where $i=\sqrt{ }-1=$, constant $A$ is the amplitude of the pressure gradient, $\kappa=\sqrt{ } i \omega \rho / \eta$, $\omega$ is angular frequency, and $I_{0}$ is the modified Bessel function of zero order [Biot, 1956].

The fuild flow carries the excess charges in the diffuse layer, resulting in the streaming current $I_{s}=2 \pi \int_{0}^{R} \rho_{e}(r) u(r) r \mathrm{~d} r$. As discussed before, the charge density $\rho_{e}(r)$ can be obtained once the electric potential $\psi(r)$ is solved from Equations (1) and (2). As it is a transcendental equation, there is no analytical solution of Equation (1). In previous studies [e.g. Pride and Morgan, 1991; Jackson and Leinov, 2012], the DebyeHückel approximation $\sinh \left(z e \psi(r) / k_{B} T\right) \approx z e \psi(r) / k_{B} T$ is used to linearize the right hand side of Equation (1),

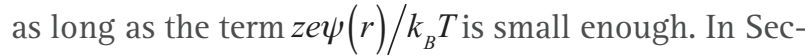
tion 3.2, we will delve deeper into the applicable condition of this linear approximation by analyzing its influences on the ECC. By solving Equations (1) and (2) with the linear approximation, the analytical solution of $\psi(r)$ and $\rho(r)$ for the cylindrical capillary are obtained as [Abramowitz and Stegun, 1964],

$$
\begin{aligned}
& \psi=\zeta I_{0}(r / d) / I_{0}(R / d) \\
& \rho_{e}=-\varepsilon \zeta I_{0}(r / d) / d^{2} I_{0}(R / d)
\end{aligned}
$$

where $d=\sqrt{\varepsilon k_{B} T / 2 n^{0} e^{2} v^{2}}$ is the Debye length characterizing the EDL thickness [Kirby and Hasselbrink, 2004].

Substituting Equations (4) and (6) into the average streaming current density $J_{s}=\frac{2}{R^{2}} \int_{0}^{R} \rho_{e}(r) u(r) r \mathrm{~d} r$, it yields

$$
J_{s}=\frac{2 \varepsilon \xi A}{\eta\left(1-d^{2} \kappa^{2}\right)}\left(\frac{I_{1}(\kappa R)}{\kappa R I_{0}(\kappa R)}-\frac{d I_{1}(R / d)}{R I_{0}(R / d)}\right) \exp (i \omega t)
$$

Thus, the streaming-current ECC $L_{12}=J_{S} / \nabla p$ in the cylindrical capillary is written as,

$$
L_{12}=\frac{-2 \varepsilon \xi}{\eta\left(1-d^{2} \kappa^{2}\right)}\left(\frac{I_{1}(\kappa R)}{\kappa R I_{0}(\kappa R)}-\frac{d I_{1}(R / d)}{R I_{0}(R / d)}\right)
$$

It is seen that Equation (8) depends on the normalized radius (the ratio of capillary radius to Debye length $R / d$ ). When $R / d \rightarrow \infty$, Equation (8) reduces to the Packard's ECC that derived under the thin EDL assumption [Packard, 1953; Reppert et al., 2001].

Note that the ECC is independent of conductivity, which is different from the streaming potential coefficient for the ratio of the streaming potential to the pressure difference [e.g. Pengra et al., 1999; Reppert et al., 2001; Tardif et al., 2011]. Dividing Equation (8) by the conductivity, it yields the streaming potential coefficient [Jouniaux et al., 2000]. For porous and fractured rocks, the conductivity is the sum of the fluid conductivity and the surface conductivity [e.g. Revil et al., 2014].

Similar to the case of the cylindrical capillary mentioned above, we can obain the ECC for the capillary slit. In this case, the governing equations changes from the axisymmetric cylindrical coordinates to the $2 \mathrm{D}$ rectangular coordinates. Thus Equation (3) without the electric field force reduces to,

$$
\rho \frac{\partial u(r)}{\partial t}=\eta \frac{\partial^{2} u(r)}{\partial r^{2}}-\frac{\partial P}{\partial z}
$$

To solve Equation (9) with $u(\mathrm{R})=0$, the flow velocity motivated by $\partial P / \partial \mathrm{z}=-A \exp (i \omega t)$ is obtained as,

$$
u(r)=\frac{i A}{\omega \rho}\left(\frac{\cosh (\kappa r)}{\cosh (\kappa R)}-1\right) \exp (i \omega t)
$$

To solve Equations (1) and (2) in the 2D rectangular coordinates, the electric potential and the charge density are respectively given by $\psi(r)=\zeta \cosh (r / d) / \cosh (R / d)$ and $\rho_{e}(r)=-\varepsilon \xi \cosh (r / d) / d^{2} \cosh (R / d)$ [Kirby and Hasselbrink, 2004]. Then the average streaming current density is written as

$$
J_{s}=\frac{\varepsilon \zeta A}{\eta\left(1-d^{2} \kappa^{2}\right)}\left(\frac{\sinh (\kappa R)}{\kappa R \cosh (\kappa R)}-\frac{d \sinh (R / d)}{R \cosh (R / d)}\right) \exp (i \omega t)(11)
$$

Thus, the streaming-current ECC for the capillary slit is given by

$$
L_{12}=\frac{-\varepsilon \xi}{\eta\left(1-d^{2} \kappa^{2}\right)}\left(\frac{\sinh (\kappa R)}{\kappa R \cosh (\kappa R)}-\frac{d \sinh (R / d)}{R \cosh (R / d)}\right)
$$

Similar to Equation (8), Equation (12) also depends on the ratio of capillary radius (half width) of capillary slit to Debye length.

\subsection{ELECTROOSMOSIS ECC}

For the electroosmosis effect driven by an applied electric field, the fluid-flow velocity is governed by Equation (3) when the second term on its right side vanishes. Suppose the sinusoidal electric field along the capillary axis is $E=A_{E} \exp (i \omega t)$, the electroosmosis-flow velocity 
$u_{E}(\mathrm{r})$ is obtained as follows, by solving Equation (3) without $\partial P / \partial \mathrm{z}$ and using Equation (6) and the boundary condition $u_{E}(\mathrm{r})=0$,

$$
u_{E}(r)=\frac{-\varepsilon \zeta A_{E}}{\eta\left(1-d^{2} \kappa^{2}\right)}\left(\frac{I_{0}(\kappa r)}{I_{0}(\kappa R)}-\frac{I_{0}(r / d)}{I_{0}(R / d)}\right) \exp (i \omega t)
$$

Substituting Equation (13) into the average flow velocity $J_{s}=\frac{2}{R^{2}} \int_{0}^{R} \rho_{e}(r) u(r) r \mathrm{~d} r$, it yields

$$
\bar{u}_{\mathrm{E}}=\frac{-2 \varepsilon \xi A_{E}}{\eta\left(1-d^{2} \kappa^{2}\right)}\left(\frac{I_{1}(\kappa R)}{\kappa R I_{0}(\kappa R)}-\frac{d I_{1}(R / d)}{R I_{0}(R / d)}\right) \exp (i \omega t)(14)
$$

Then the electroosmosis ECC $L_{21}=\bar{u}_{\mathrm{E}} / E$ is written as

$$
L_{21}=\frac{-2 \varepsilon \zeta}{\eta\left(1-d^{2} \kappa^{2}\right)}\left(\frac{I_{1}(\kappa R)}{\kappa R I_{0}(\kappa R)}-\frac{d I_{1}(R / d)}{R I_{0}(R / d)}\right)
$$

By comparison, it is seen that Equations (8) and (15) are identical, that is $L_{12}=L_{21}$. This indicates the correctness of our derivations.

Moreover, we prove that the Onsager reciprocity [Onsager, 1931] of the frequency-dependent ECC is still available for arbitrary scale, although it has been verified under the thin EDL assumption by Pride [1994]. When $\omega \rightarrow 0$, Equations (8) and (15) is simplified as

$$
L_{0}=\frac{-\varepsilon \xi}{\eta}\left(1-\frac{2 d}{R} \frac{I_{1}(R / d)}{I_{0}(R / d)}\right)
$$

which is the static ECC for the cylindrical capillary. When $R / d \rightarrow \infty$, Equation (16) degenerates into the $\mathrm{H}-\mathrm{S}$ equation [Jouniaux and Bordes, 2012].

For the capillary slit, the electroosmosis-flow velocity $u_{E}(\mathrm{r})$ is governed by

$$
\rho \frac{\mathrm{d} u(r)}{\mathrm{d} t}=\eta \frac{\mathrm{d}^{2} u(r)}{\mathrm{d} r^{2}}+\rho_{e}(r) E
$$

Using the same method in cylindrical capillary, the electroosmosis-flow velocity and thus the electroosmosis ECC for the capillary slit are obtained as

$$
\begin{gathered}
u_{E}(r)=\frac{-\varepsilon \varsigma A_{E}}{\eta\left(1-d^{2} \kappa^{2}\right)}\left(\frac{\cosh (\kappa x)}{\cosh (\kappa R)}-\frac{\cosh (x / d)}{\cosh (R / d)}\right) \exp (i \omega t) \text { (18) } \\
L_{21}=\frac{-\varepsilon \zeta}{\eta\left(1-d^{2} \kappa^{2}\right)}\left(\frac{\sinh (\kappa R)}{\kappa R \cosh (\kappa R)}-\frac{d \sinh (R / d)}{R \cosh (R / d)}\right)
\end{gathered}
$$

Equations (12) and (19) are also identical, which indicates that the Onsager reciprocity is still available for the capillary slit. Because it has been verified for the two extreme shapes, we deduce that the Onsager reciprocity is available for capillaries in any shape.

When $\omega \rightarrow 0$, Equations (12) and (19) is simplified as

$$
L_{0}=\frac{-\varepsilon \xi}{\eta}\left(1-\frac{d \sinh (R / d)}{\mathrm{R} \cosh (R / d)}\right)
$$

which is the static ECC for the capillary slit.

When $R / d \rightarrow \infty$, Equation (20) degenerates into the $\mathrm{H}-\mathrm{S}$ equation, which is the same as that of Equation (16).

The above-derived ECC formulas for capillaries can be extended to fluid-saturated porous media. When pore channels are regarded as tortuous capillary bundles, Equations (15) and (19) can be rewritten straightforwardly into

$$
\begin{gathered}
L=\frac{-2 \varepsilon \xi}{F \eta\left(1-d^{2} \kappa^{2}\right)}\left(\frac{I_{1}(\kappa R)}{\kappa R I_{0}(\kappa R)}-\frac{d I_{1}(R / d)}{R I_{0}(R / d)}\right) \\
L=\frac{-\varepsilon \xi}{F \eta\left(1-d^{2} \kappa^{2}\right)}\left(\frac{\sinh (\kappa R)}{\kappa R \cosh (\kappa R)}-\frac{d \sinh (R / d)}{R \cosh (R / d)}\right)
\end{gathered}
$$

where $F=\tau / \phi$ is the formation factor. Equation (21) originating from the ECC for the cylindrical capillary is applicable to the porous media with circular apertures. Equation (22) originating from the ECC for the capillary slit is applicable to the porous media with flat cracks.

\section{ANALYSIS}

\subsection{ANALYTICAL RESULTS}

In this subsection, we analyze the characteristics of the static and frequency-dependent ECCs and give comparisons with the Packard's model. Without special explanations, we employ the kelvin temperature $T=298 \mathrm{~K}\left(25^{\circ} \mathrm{C}\right)$, ionic valence $v=1$, salinity $C_{f}=0.001 \mathrm{~mol} / \mathrm{L}$ (the ionic number is calculated by $n^{0}=1000 N_{a} C_{f}$ where $N_{a}=6.02 \cdot 10^{23}$ is Avogadro constant), density $\rho=1000 \mathrm{~kg} / \mathrm{m}^{3}$, viscosity $\eta=0.001 \mathrm{~Pa} \cdot \mathrm{s}$ and dielectric permittivity $\varepsilon=80 \varepsilon_{0}\left(\varepsilon_{0}=8.85 \cdot 10^{-12} \mathrm{~F} / \mathrm{m}\right.$ is the vacuum dielectric constant) of the pore fuild.

According to these parameters, the Debye length $d=9.72 \cdot 10^{-9} \mathrm{~m}$.

Figure 2 shows the variations of the static ECCs with the normalized radius $(R / d)$ for both the two differentshaped capillaries. The longitudinal coordinate is normalized with respect to the ECC with the thin EDL assumption. It is seen from Figure 2(a) that the normalized ECCs for cylindrical capillary and capillary slit monotonically increase with the increase of $R / d$ and gradually tends to 1.0. It indicates that the static ECCs presented in this study tend to the ECC with the thin EDL assumption. when $R / d=20$ and $R / d=60$ respectively, the static ECCs for the two different capillaries are about 90\% and 95\% of the one in the thin EDL assumption. This result approaches to those given by Jackson and Leinov [2012] and Fiorentino et al., [2016], where the 


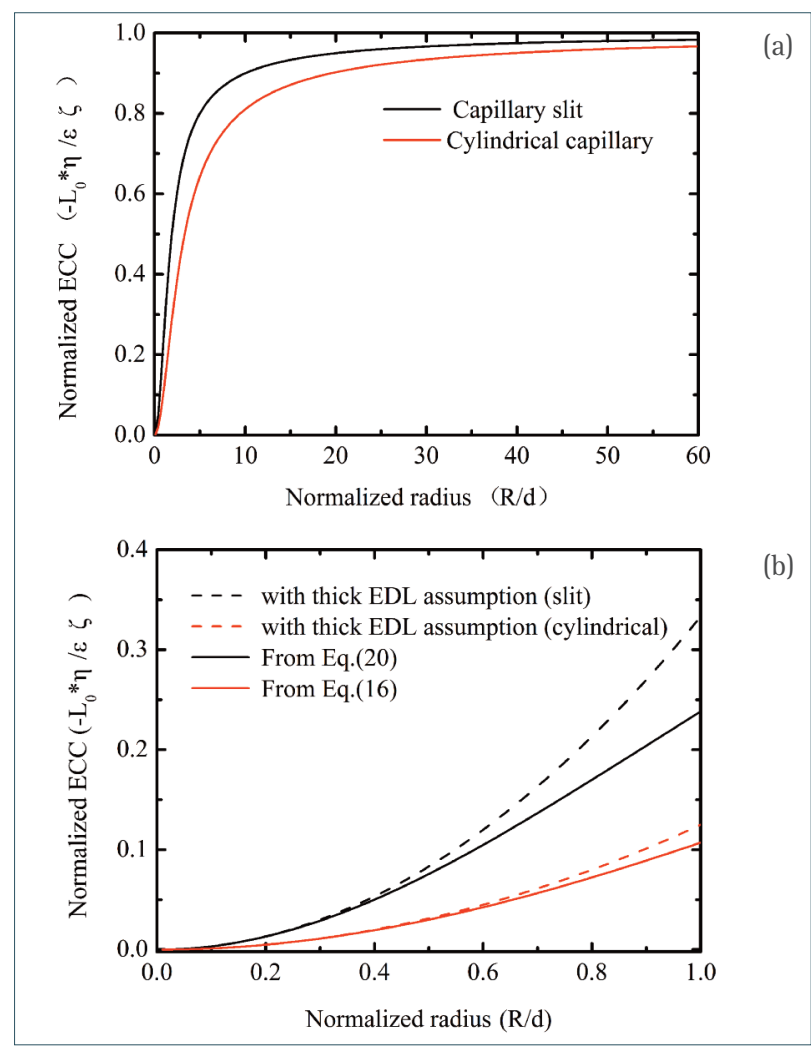

FIGURE 2. Variations of the static ECCs with the normalized radius $R / d$ for the cylindrical capillary (red solid line) and the capillary slit (black solid line). (a) $0<R / d<60$ (b) Comparisons to those with the thick EDL assumption (dashed lines) until $R / d=1$.

application conditions of the thin EDL assumption is $R / d>24$ and $R / d>52$, respectively. Thus it confirms that the thin EDL assumption is applicable if the capillary radius is several ten times of the Debye length or more.

In Figure 2(a), when $R / d$ is about less than 10 , the normalized ECCs start to decrease significantly with the decrease of $R / d$. As a result, the discrepancy between our static ECCs and that under the thin EDL assumption increases significantly, while our static ECCs gradually tend to the ECCs under the thick EDL assumption. Figure 2(b) gives comparisons between the static ECCs in this study and the ones based on the thick EDL assumption. It is found that the ECCs under the thick EDL assumption overestimate the static ECCs. This overestimation increases with $\mathrm{R} / \mathrm{d}$. When the overestimation is up to $10 \%$, the $R / d$ is calculated about 0.5 and 0.78 for the cylindrical capillary and the capillary slit, respectively. This basically agrees with Jackson and Leinov [2012] where the thick EDL assumption is applicable when $R / d<0.81$. Besides, the static ECC for the capillary slit is larger than that for the cylindrical capillary. The reason is that the interface of the capillary slit is larger than that of the cylindrical capillary with the same cross-sectional area. Thus the average flow velocity and then the ECC of the former are higher than that of the latter.

Figure 3 shows the variations of the frequency-dependent ECC with frequency. The solid line denotes the variation in amplitude, while the dashed line denotes the variation in phase. The three panels on the left are for the cylindrical capillaries with $R / d=0.6,10$ and 50 from top to bottom, while the three ones on the right are for the capillary slits. For $R / d=0.6$, the thick EDL assumption is applicable, while for $R / d=50$, the thin EDL assumption is applicable. For $R / d=10$, however, neither of the two assumptions are reasonable. It is seen that the amplitudes as well as the phases have same variation trendencies for the capillaries with different radii or cross-sectional shapes. The amplitude decreases smoothly (basically unchanged) starting from the static ECC first, then decreases sharply until the inflexion point, and finally decreases smoothly to zero. As mentioned above, the capillaries with different radii or cross-sectional shapes have different static-ECC values. The frequency corresponding to the inflexion point increases with the decrease of $R / d$. In the upper two panels for $R / d=0.6$, this frequency is on the order of $10 \mathrm{GHz}$. Such a high frequency is far out of the frequency range in exploration geophysics. Nevertheless, the frequency of interest may be up to this order in the fields of nondestructive examination, bio-MEMS and so on.

The ECC phase reflects the response delay between the mechanical and electromagnetic fields of the timevarying electrokinetic effects. As shown in Figure 3, the phase increases from zero with frequency. It increases smoothly first at low frequencies, then increases sharply until the inflexion point, and finally increases smoothly to the high-frequency-limit value after the inflexion point. It is found that this high-frequency limit phase increases with the decrease of $R / d$. This characteristic is different from that of the Packard's ECC, which is independent of the capillary radius and remains at $45^{\circ}$. In Figure 4, we compare the variations of the high-frequency-limit phase with $R / d$ between the two different shaped capillaries. The results are identical with each other at any radius, indicating that the high-frequency limit phase is independent of the cross-sectional shape of the capillary. With the decrease of $R / d$, the high-frequency limit phase increases from $45^{\circ}$ to $90^{\circ}$.

Figure 4 is divided into three regions according to the applicable conditions of the thin and thick EDL assumptions mentioned above. The two shadow regions $(R / d<0.81$ and $R / d>24)$ denote the scales that the thick and thin EDL assumptions are applicable, respectively, while the white area $(0.81<R / d<24)$ denotes the scale between them. For the two scales of $R / d<0.81$ and 


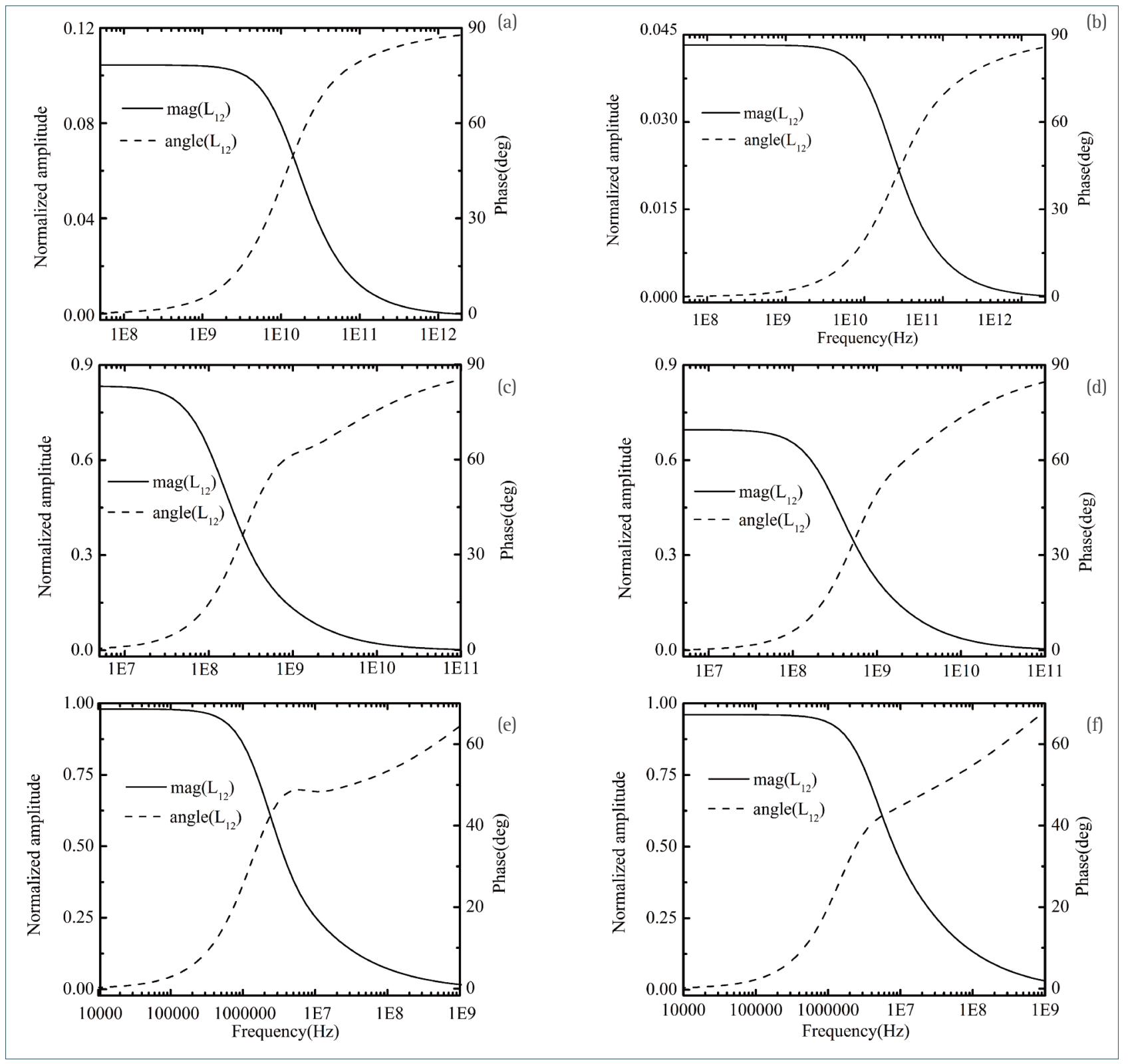

FIGURE 3. Amplitudes and phases of the frequency-dependent ECC. Panels (a), (c) and (e) are for the capillary slits with $R / d=0.6,5$ and 50, respectively. Panels (b), (d) and (f) are for the cylindrical capillaries with $R / d=0.6,5$ and 50 , respectively.

$0.81<R / d<24$, the high-frequency-limit phase basically keeps at $90^{\circ}$. For $R / d>24$, however, it decreases from $90^{\circ}$ to $45^{\circ}$ with the increase of $R / d$. Until $R / d>10^{5}$, it approaches to $45^{\circ}$, which is the high-frequency-limit phase of the Packard's ECC under the thin EDL assumption. Thus if the high-frequency-limit phase is considered, obviously a higher $R / d$ than that for the static ECC is necessary to the thin EDL assumption.

In Figure 5, we compare the real and imaginary parts of the ECC presented in this study with those of the Packard's ECC for different $R / d$. It is seen that the discrepancies in both the real and imaginary parts increase with $R / d$. By comparison, the discrepancy of the real part is relatively larger than that of the imag- inary part, especially at low frequencies. The reason can be found from the ECC formulas derived in this study. The two terms $d \mathrm{I}_{1}(R / d) / R I_{0}(R / d)$ and $1-d^{2}-\kappa^{2}$ in Equation (8) reflect the modification to the Packard's model. The first term is independent of the imaginary part, while the second term is negligible at low frequencies. Besides, the maximums of the imaginary part for the two models correspond to the same frequency, which is referred to as the transition frequency that separates the low-frequency viscous flow from the high frequency inertial flow [Tardif et al., 2011]. For cylindrical capillaries and capillary slits, the transition frequencies are expressed by $\omega t=8 \eta / \rho R^{2}$ and $\omega t=3 \eta / \rho R^{2}$, respectively, which are independent of the EDL and the electric characteristics. 


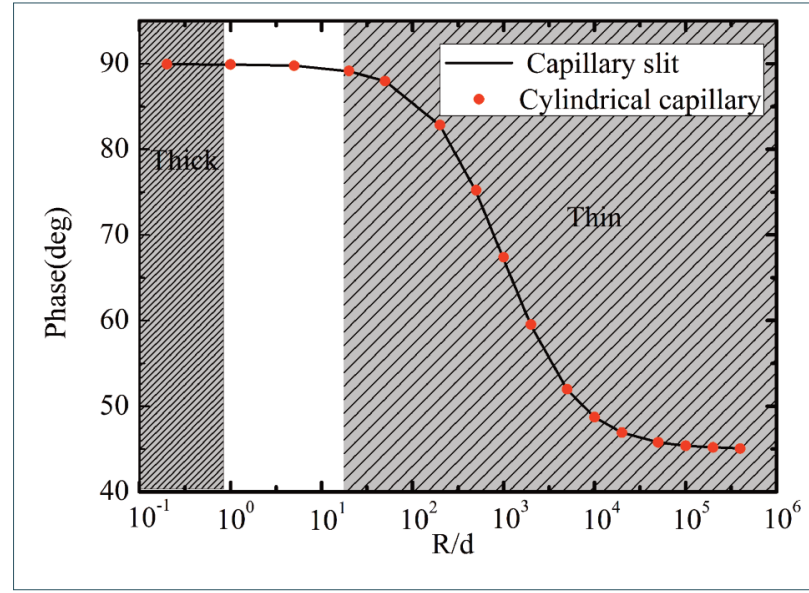

FIGURE 4. Variations of the high-frequency limit phase with the normalized radius $R / d$ for the cylindrical capillary (red dotted line) and the capillary slit (black solid line). The horizontal axis is separated into the three regions of $R / d<0.81,0.81<R / d<24$ and $R / d>24$.

\subsection{NUMERICAL SOLUTIONS}

As stated above, the approximation $\sinh \left(v e \psi / k_{B} T\right) \approx v e \psi / k_{B} T$ has been used to obtain the analytic solution of the Poisson-Boltzmann equation [Pengra et al., 1999; Reppert et al., 2001]. This approximation is mathematically valid if the term ve $\psi / k_{B} T$ is less than 1.0. Thus it is applicable if the absolute value of the zeta potential (the maximum of $\psi(r)$ is less than $25 \mathrm{mV}$ when $v=1$ and $T=298 \mathrm{~K}\left(25^{\circ} \mathrm{C}\right)$ [Pride, 1994]. Nevertheless, experimental measurements show that there are many porous rocks whose $|\xi|$ is much larger than $25 \mathrm{mV}$ and even reaches to $200 \mathrm{mV}$, meanwhile, their ionic valence $v$ is possiable to be larger than 1.0 [Glover et al., 2012]. In these cases, the approximation $\sinh \left(v e \psi / k_{B} T\right) \approx v e \psi / k_{B} T$ is inapplicable becasue $v e \psi / k_{B} T$ is larger than 1.0. Although alternative approximations such as $\sinh \left(v e \psi / k_{B} T\right) \approx \exp \left(v e \psi / k_{B} T\right) / 2$ can be used if $v e \psi / k_{B} T$ is larger than 1.0 [Levine et al., $1975]$, it is inconvenient in practice to employ piecewise ECC formulas for ve $\psi / k_{B} T>1.0$ and $v e \psi / k_{B} T<1.0$, respectively.

Recently, Fiorentino et al. [2016, 2017] numerically solved the Poisson-Boltzmann equation without either of the approximations mentioned above. They analyzed the effects of the linear approximation on the electric potential and the static streaming potential coefficient. In this subsection, however, we investigate the effects on the static and frequency-dependent ECCs as well as the electric potential distribution. We also numerically solve the Poisson-Boltzmann equation without any approximation and compare the difference between the numerical solutions and the analytical solutions with the linear approximation. In view of the similar characteristics of the ECC for capillaries with different cross-



FIGURE 5. Comparisons of the present frequency-dependent ECC (solid line) with Packard's model (dotted line) for three different radii of 1000 (black), 50 (red) and 10 (blue) times of the Debye length respectively.

sectional shapes, we only consider the case of capillary slits below.

In Figure 6, we compare the electric potential distribution and the frequency-dependent ECC between the numerical and analytical solutions. The zeta potential $\zeta=-60 \mathrm{mV}$ is employed. By calculation, the maximums of the term ve $\psi / k_{B} T$ and $\sinh \left(v e \psi / k_{B} T\right)$ are 2.33 and 5.10 , respectively. Thus, the linear approximation cannot be adopted. Figures 6(a) and 6(b) are the comparisons for $R / d=0.62(\mathrm{R}=6 \mathrm{~nm})$, which show obvious discrepancies between the two solutions. In Figure 6(a), the potential difference of the numerical solution from the shear-slip surface to the capillary center is $15 \mathrm{mV}$ (from $-45 \mathrm{mV}$ to $-60 \mathrm{mV}$ ), which is larger than the analytical solution. This result is similar to that of Figure 11(a) in semi-log scale given by Fiorentino et al. [2016]. It means that the electric charges calculated by the numerical method are larger than that of the analytical method, resulting in a larger electrokinetic conversion. As a result, the real and imaginary parts of the numerical solutions for the ECC are significantly larger than those of the analytical solutions as shown in Figure 6(b). Figures $6(\mathrm{c})$ and $6(\mathrm{~d})$ are the comparisons for $R / d=10.3$ $(\mathrm{R}=100 \mathrm{~nm})$, in which the discrepancies are extremely small. This indicates that the discrepancies decrease with the increase of $R / d$. When $R / d$ is large enough, the effect of the linear approximation on the ECC is negligible although it is already mathematically invalid.

In Figure7, we compare the electric potential and ECC for different zeta potential with $R / d=10.3$. Figures 7 (a) and 7(b) are the comparisons for $\zeta=-100 \mathrm{mV}$, while Figures $7(\mathrm{c})$ and $7(\mathrm{~d})$ are those for $\zeta=-200 \mathrm{mV}$. It is shown that the discrepancies in both the electric potential and ECC increase with the increase of $|\xi|$. From 




FIGURE 6. Comparisons of the results between the numerical (black line) and the analytical (red line) solutions for $\zeta=-60 \mathrm{mV}$. (a) and (b) are the electric potential distribution and the frequency-dependent ECC for $R / d=0.62(\mathrm{R}=6 \mathrm{~nm})$, respectively, while (c) and (d) are those for $R / d=10.3(\mathrm{R}=100 \mathrm{~nm})$.

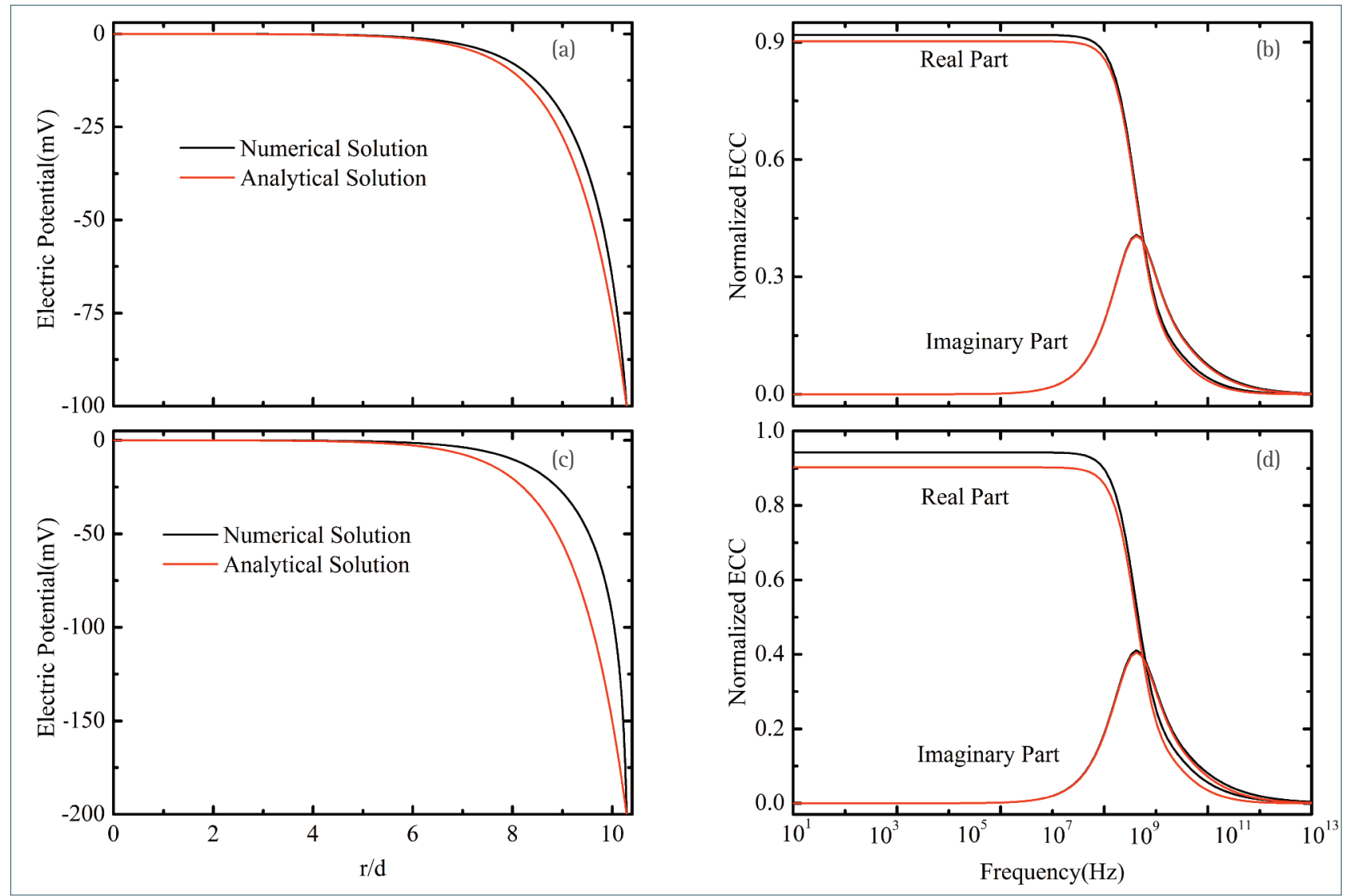

FIGURE 7. Comparisons of the results between the numerical (black line) and the analytical (red line) solutions for $R / d=10.3$ ( $\mathrm{R}=100$ $\mathrm{nm}$ ). (a) and (b) are the electric potential distribution and the frequency-dependent ECC for $\xi=-100 \mathrm{mV}$, respectively, while (c) and (d) are those for $\xi=-200 \mathrm{mV}$. 


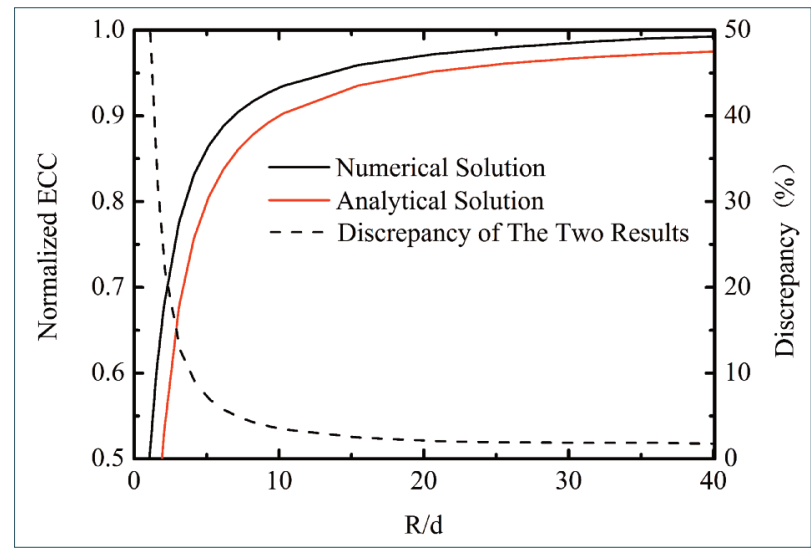

FIGURE 8. Comparison between the numerical (black solid line) and the analytical (red solid line) solutions of the variation of static ECC with $R / d$ for $\xi=-150 \mathrm{mV}$. The discrepancy between the two solutions is also given by the black dashed line.

Figure 7(d) for $\zeta=-200 \mathrm{mV}$, it is seen that the discrepancy of the ECC's real-part is only about 5.0\% and that of the ECC's imaginary-part is much smaller. Among the estimated zeta-potential for 269 samples given by Glover et al. [2012], only three of them exceed $-150 \mathrm{mV}$ and only one exceeds $-200 \mathrm{mV}$. Thus, the linear approximation is applicable in practice when the salinity is higher than $0.001 \mathrm{~mol} / \mathrm{L}$, except for capillaries with extremely small radii. Since the effect of the linear approximation on the ECC's real part is relatively larger at low frequencies, Figure8 shows the discrepancy of the static ECCs between the numerical and analytical methods with different $R / d$. The dash line denotes the discrepancy between the two results. It is seen that the discrepancy is less than $5.0 \%$ for about $R / d>7$ and $\zeta=-150 \mathrm{mV}$, and increases significantly with the decrease of $R / d$.

\section{CONCLUSIONS}

In this study, we have theoretically derived the frequency-dependent ECC with dependence on the normalized radius $R / d$ (the ratio of the capillary/pore radius to the Debye length). When compared with previous studies, the present ECC can be used with arbitrary capillary/pore scale, and thus is not restricted to the thin or thick EDL assumption. We have considered two different cross-sectional shapes, which are the cylindrical capillary and the capillary slit, thus these ECC formula can be extended to porous media with circular apertures and flat fractures, respectively. We have proved that the Onsager reciprocity of the streaming current and electroosmosis ECCs is satisfied for arbitrary capillary/pore scales and cross-sectional shapes.
According to these ECC formulas, we have calculated the applicable conditions of the thin and thick EDL assumptions, which are $R / d>20$ and $R / d<0.8$, respectively. For realistic rocks, the thin EDL assumption may be inapplicable to sandstone with low salinities and the thick EDL assumption may be inapplicable to mudstone and shale with high salinities. The results also show that the ECC of flat fractures is always larger than that of circular apertures with identical pore scales and the highfrequency limit phase of the ECC does not remain at $45^{\circ}$ as the Packard model, but increases monotonically from $45^{\circ}$ to $90^{\circ}$ with the decrease of the normalized radius. By numerically solving the Poisson-Boltzmann equation, it is found that the effects of the linear approximation on the electric potential distribution and the ECC increase with the decrease of the normalized radius. Even if the linear approximation has already been mathematically invalid, it is still applicable to realistic rocks except for $R / d<7$ with $|\xi|$ larger than $150 \mathrm{mV}$.

Acknowledgements. The authors are very grateful to two reviewers for their suggestions. This work is jointly supported by National Natural Science Foundations of China $(41574112,41674121$ and 11734017), the National Science and Technology Major Project of China (2017ZX05019006-006) and the Natural Science Foundation of Heilongjiang Province of China (D2015001).

\section{REFERENCES}

Abramowitz M, Stegun 1 A. (1964), Handbook of mathematical functions: with formulas, graphs, and mathematical tables. Courier Corporation.

Allègre V., Lehmann F., Ackerer P., Jouniaux L., Sailhac P. (2012), A 1-D modelling of streaming potential dependence on water content during drainage experiment in sand, Geophys. J. Int., 189(1), 285-295.

Biot M A. (1956), Theory of propagation of elastic waves in a fluid saturated porous solid. II. Higher frequency range. J. Acoust. Soc. Am., 28(2): 179-191.

Bordes C., Jouniaux L., Garambois S., Dietrich M., Pozzi J.P., Gaffet S. (2008), Evidence of the theoretically predicted seismo-magnetic conversion, Geophys. J. Int., 174(2), 489-504.

Burgreen D., Nakache F.R. (1964), Electrokinetic flow in ultrafine capillary slits, J. Phys. Chem., 68(5), 1084-1091.

Cases J.M. (1969), Point de charge nulle et structure des silicates, J. Chim. Phys., 66, 1602-1611.

DesRoches A.J., Butler K.E. (2016), Monitoring and modelling of pumping-induced self-potentials for transmissivity estimation within a heterogeneous 
confined aquifer, Geophys. J. Int., 207(3), 17221738.

Dupuis J.C., Butler K.E., Kepic A.W., Harris B.D. (2009), Anatomy of a seismoelectric conversion: Measurements and conceptual modeling in boreholes penetrating a sandy aquifer, J. Geophys. Res., 114(B10), B10306.

Fiorentino E.A., Toussaint R., Jouniaux L. (2016), Lattice Boltzmann modelling of streaming potentials: variations with salinity in monophasic conditions, Geophys. J. Int., 205(1), 648-664.

Fiorentino E.A., Toussaint R., Jouniaux L. (2017), Twophase Lattice Boltzmann modelling of streaming potentials: influence of the air-water interface on the electrokinetic coupling, Geophys. J. Int, 208(2), 1139-1156.

Gao Y., Chen X., Hu H., Zhang J. (2013), Early electromagnetic waves from earthquake rupturing: 1 . theoretical formulations, Geophys. J. Int. 192(3), 1288-1307.

Gao Y., Harris J.M., Wen J., Huang Y., Twardzik C., Chen X., Hu H. (2016), Modeling of the coseismic electromagnetic fields observed during the $2004 \mathrm{Mw}$ 6.0 Parkfield earthquake, Geophys. Res. Lett., 43(2), 620-627.

Gaudin A.M., Fuerstenau D.W. (1955), Streaming Potential Studies. Quartz Flotation with Cationic Collectors, Trans. AlME, 202, 958.

Gierst L. (1966), Double Layer and Electrode Kinetics, J. Am. Chem. Soc., 88(20), 4768.

Glover P.W., Walker E. (2008), Grain-size to effective poresize transformation derived from electrokinetic theory, Geophysics, 74(1), E17-E29.

Glover P.W., Déry N. (2010), Streaming potential coupling coefficient of quartz glass bead packs: Dependence on grain diameter, pore size, and pore throat radius, Geophysics, 75(6), F225-F241.

Glover P.W., Walker E., Jackson M.D., (2012), Streamingpotential coefficient of reservoir rock: A theoretical model, Geophysics, 77(2), D17-D43.

Gouy M. (1910), Sur la constitution de la charge électrique à la surface d'un electrolyte, J. Phys. Theor. Appl., 9(1), 457-468.

Guan W., Hu H., Wang Z. (2013), Permeability inversion from low-frequency seismoelectric logs in fluid-saturated porous formations, Geophys. Prospect., 61(1), 120-133.

Guan W, Yin C, Wang J, et al. (2015), Theoretical study on the amplitude ratio of the seismoelectric field to the Stoneley wave and the formation tortuosity estimation from seismoelectric logs. Geophys. J. Int., 203(3), 2277-2286.
Hu H, Wang K, Wang J. (2000), Simulation of an acoustically induced electromagnetic field in a borehole embedded in a porous formation. Massachusetts Institute of Technology. Earth Resources Laboratory.

$\mathrm{Hu} \mathrm{H}$, Liu J. (2002), Simulation of the converted electric field during acoustoelectric logging, SEG Technical Program Expanded Abstracts 2002. Society of Exploration Geophysicists, 348-351.

Ishido T., Mizutani H. (1981), Experimental and theoretical basis of electrokinetic phenomena in rock water systems and its applications to geophysics, J. Geophys. Res., 86(B3), 1763-1775.

Jackson M.D., Leinov E. (2012), On the validity of the "thin" and "thick" double-layer assumptions when calculating streaming currents in porous media, Int. J. Geophys., 2012, 897807.

Jouniaux L., Bernard M.L., Zamora M., Pozzi J.P. (2000), Streaming potential in volcanic rocks from Mount, J. Geophys. Res., 105(B4), 8391-8401.

Jouniaux L., Bordes C. (2012), Frequency-dependent streaming potentials: a review, Int. J. Geophys., 2012, 648781.

Jouniaux L., Zyserman F. (2016), A review on electrokinetically induced seismo-electrics, electro-seismics, and seismo-magnetics for Earth sciences, Solid Earth, 7(1), 249.

Kirby B.J., Hasselbrink E.F. (2004), Zeta potential of microfluidic substrates: 1 . Theory, experimental techniques, and effects on separations, Electrophoresis, 25(2), 187-202.

Kröger B., Yaramanci U., Kemna A. (2014), Numerical analysis of seismoelectric wave propagation in spatially confined geological units. Geophys. Prospect., 62(1), 133-147.

Levine S., Marriott J.R., Neale G., Epstein N. (1975), Theory of electrokinetic flow in fine cylindrical capillaries at high zeta-potentials, J. Colloid and Interf. Sci., 52(1), 136-149.

Li H.C., De Bruyn P. L. (1966), Electrokinetic and adsorption studies on quartz, Surf. Sci., 5(2), 203-220.

Li S X, Pengra D B, Wong P. (1995), Onsager's reciprocal relation and the hydraulic permeability of porous media, Phys. Rev. E, 51(6), 5748.

Mikhailov O.V., Queen J., Toksöz M.N. (2000), Using borehole electroseismic measurements to detect and characterize fractured (permeable) zones, Geophysics, 65(4), 1098-1112.

Monetti A., Troiano A., Di Giuseppe M.G., et al. (2014) Modeling Self-Potential Effects During Reservoir Stimulation in Enhanced Geothermal Systems. Excerpt from the Proceedings of the 2014 COMSOL Conference in Cambridge 
Onsager L. (1931), Reciprocal Relations in Irreversible Processes. 1., Phys. Rev. 37(4), 405-426.

Ozaki Y., Mikada H, Goto T., et al. (2014), Self-potential inversion for the estimation of permeability structure, J. Environ. Eng. Geoph., 19(3), 193-199.

Packard R.G. (1953), Streaming potentials across glass capillaries for sinusoidal pressure, J. Chem. Phys., 21(2), 303-307.

Pengra D.B., Xi Li S., Wong P. (1999), Determination of rock properties by low-frequency AC electrokinetics, J. Geophys. Res., 104(B12), 29485-29508.

Pride S.R. (1994), Governing equations for the coupled electromagnetics and acoustics of porous media, Phys. Rev. B, 50(21), 15678-15696.

Pride S.R., Berryman J.G., Harris J.M. (2004), Seismic attenuation due to wave-induced flow, J. Geophys. Res., 109(B1), B01201.

Pride S.R., Morgan F.D. (1991), Electrokinetic dissipation induced by seismic waves, Geophysics, 56(7), 914-925.

Quincke G. (1861), Ueber die Fortführung materieller Theilchen durch strömende Elektricität, Ann.PhysBerlin, 189(8), 513-598.

Rangarajan R., Muralidharan D., Chandra S., et al. (2014), Time lapse tracer and SP measurements to characterize the hydrodynamics of fractured granite aquifer: A case study, J. Geol. Soc. India, 83(6), 681687.

Reppert P.M., Morgan F.D. (2003), Temperature-dependent streaming potentials: 1. Theory, J. Geophys. Res., 108(B11), 2546.

Reppert P.M., Morgan F.D., Lesmes D.P., Jouniaux L. (2001), Frequency-dependent streaming potentials, J. Colloid and Interf. Sci., 234(1), 194-203.

Revil A., Kessouri P., Torres-Verdín C. (2014), Electrical conductivity, induced polarization, and permeability of the Fontainebleau sandstone, Geophysics, 79(5), D301-D318.

Revil A., Mahardika H. (2013), Coupled hydromechanical and electromagnetic disturbances in unsaturated porous materials, Water resour. Res., 49(2), 744-766.

Rice C.L., Whitehead R. (1965), Electrokinetic flow in a narrow cylindrical capillary, J. Phys. Chem., 69(11), 4017-4024.

Sava P., Revil, A. 2012. Virtual electrode current injection using seismic focusing and seismoelectric conversion, Geophys. J. Int., 191(3), 1205-1209.

Smoluchowski M. (1905), Zur theorie der elektrischen kataphorese und der oberflchenleitung, Physikalische Z., 6, 529-531.

Somasundaran P., Kulkarni R.D. (1973), A new streaming potential apparatus and study of temperature effects using it, J. Colloid Interf. Sci., 45(3), 591-600.
Tardif E., Glover P.W., Ruel J. (2011), Frequency-dependent streaming potential of Ottawa sand, J. Geophys. Res., 116, B04206.

Thomson W.T. (1996), Theory of vibration with applications, Fourth edition, CRC Press.

Von Helmholtz H.L.F. (1879), Studien über electrische Grenzschichten, Ann. Phys-Berlin, 243(7), 337-382.

Walker E., Glover P.W. (2010), Permeability models of porous media: Characteristic length scales, scaling constants and time-dependent electrokinetic coupling, Geophysics, 75(6), E235-E246.

Wang J., Hu H., Guan W., Li H. (2015), Electrokinetic experimental study on saturated rock samples: zeta potential and surface conductance, Geophys. J. Int., 201(2), 869-877.

Warden S., Garambois S., Jouniaux L., et al. (2013), Seismoelectric wave propagation numerical modelling in partially saturated materials, Geophys. J. Int., 194(3): 1498-1513.

Wiedemann G. (1852), Ueber die Bewegung von Flüssigkeiten im Kreise der geschlossenen galvanischen Säule, Annalen der Physik, 163(11), 321-352.

Zyserman F.1., Jouniaux L., Warden S., Garambois S. (2015), Borehole seismoelectric logging using a shear-wave source: Possible application to CO2 disposal?, Int. J. Greenh. Gas Con., 33, 89-102

*CORRESPONDING AUTHOR: Wei GUAN, Department of Mechanics, Harbin Institute of Technology, Harbin, P. R. China email: guanw@hit.edu.cn (c) 2018 the Istituto Nazionale di Geofisica e Vulcanologia. All rights reserved. 